\title{
Distribución, parámetros poblacionales y dieta de Astropecten marginatus (Asteroidea: Astropectinidae) en el Atlántico venezolano
}

\author{
Ileana Ortega, Alberto Martín \& Yusbelly Díaz \\ Universidad Simón Bolívar. Departamento de Estudios Ambientales. Centro de Biodiversidad Marina. Laboratorio \\ de Crustáceos Peracáridos. Apdo. 89000. Caracas 1086-A, Venezuela; ileanaortega@gmail.com, amartinz@usb.ve, \\ yusdiaz@usb.ve
}

Recibido 17-IV-2009. Corregido 19-VII-2010. Aceptado 19-VIII-2010.

\begin{abstract}
Distribution, population parameters, and diet of Astropecten marginatus (Asteroidea: Astropectinidae) in the Venezuelan Atlantic coast. Astropecten marginatus is a sea star widely distributed in Northern and Eastern South America, found on sandy and muddy bottoms, in shallow and deep waters. To describe some of its ecological characteristics, we calculated it spatial-temporal distribution, population parameters (based on size and weight) and diet in the Orinoco Delta ecoregion (Venezuela). The ecoregion was divided in three sections: Golfo de Paria, Boca de Serpiente and Plataforma Deltana. Samples for the rainy and dry seasons came from megabenthos surveys of the "Línea Base Ambiental Plataforma Deltana (LBAPD)" and "Corocoro Fase I (CFI)" projects. The collected sea stars were measured, weighted and dissected by the oral side to extract their stomach and identify the preys consumed. A total of 570 sea stars were collected in LBAPD project and 306 in CFI one. The highest densities were found during the dry season in almost all sections. In LBAPD project the highest density was in "Plataforma Deltana" section $\left(0.007 \pm 0.022 \mathrm{ind} / \mathrm{m}^{2}\right.$ in dry season and $0.014 \pm 0.06 \mathrm{ind} / \mathrm{m}^{2}$ in rainy season) and in the CFI project the densities in "Golfo de Paria" section were $0.705 \pm 0.829 \mathrm{ind} / \mathrm{m}^{2}$ in rainy season and $1.027 \pm 1.107 \mathrm{ind} / \mathrm{m}^{2}$ in dry season. The most frequent size range was $3.1-4.6 \mathrm{~cm}$. The highest biomass was found in "Golfo de Paria" section $\left(7.581 \pm 0.018 \mathrm{mg} / \mathrm{m}^{2}\right.$ in dry season and $0.005 \pm 6.542 \times 10^{-06} \mathrm{mg} / \mathrm{m}^{2}$ in rainy season for $2004-2005$ and $3.979 \pm 4.024 \mathrm{mg} / \mathrm{m}^{2}$ in dry season; and $3.117 \pm 3.137 \mathrm{mg} / \mathrm{m}^{2}$ in rainy season for 2006). A linear relationship was found between the sea star size and its weight but no relationship was observed between its size and the depth where it was collected. Mollusks are dominant in the sea star diet (47.4\% in abundance). The diet in any of the sections, seasons or between projects or size class was heterogeneous, using multivariate ordinations (MDS) and SIMPER analysis and there was no difference in the prey number or food elements that a sea star can eat. Although A. marginatus has been described as a predator, in this study were also inferred scavenger and detritivorous habits. Rev. Biol. Trop. 59 (1): 53-69. Epub 2011 March 01.
\end{abstract}

Key words: Astropecten marginatus, Echinodermata, Astropectinidae, Venezuela, megabenthos, diet, distribution.

Astropecten marginatus Gray 1840 es una estrella de mar perteneciente a la Clase Asteroidea, Orden Paxillosida y Familia Astropectinidae. El género Astropecten está distribuido en aguas tropicales y subtropicales; habitando en su mayoría en sustratos arenosos y arenofangosos (Lera 1980, Bitter \& Penchaszadeh 1983, Bitter 2000).

La distribución de A. marginatus es muy amplia, habiéndose reportado desde el Mar Caribe (Puerto Rico, República Dominicana,
Colombia, Venezuela) hasta Brasil (Rio Grande do Sul) (Kenk 1944, Herrera-Moreno \& Betancourt 2004, Benavides-Serrato et al. 2005, Vala 2005). Dentro de Venezuela ha sido encontrada en los estados Zulia, Falcón, Carabobo, Nueva Esparta, Sucre y Delta Amacuro (Zoppi de Roa 1967, Lera 1980, Martín et al. 2007). Este equinodermo puede encontrarse tanto en aguas someras como profundas (1 y 130m de profundidad) (Clark \& Downey 1992, Vala 2005). 
Las estrellas del género Astropecten presentan digestión intraoral y no evierten el estómago como la mayoría de los asteroideos, careciendo de mecanismos para triturar su alimento, por lo que deben ingerir sus presas enteras y digerirlas internamente. Esta característica permite que se pueda conocer su dieta analizando el contenido estomacal del individuo (Lera 1980, Bitter \& Penchaszadeh 1983, Vala 2005, Caregnato et al. 2009).

Muchos asteroideos han sido catalogados como carnívoros, y su depredación juega un papel importante en la estructura y función de las comunidades marinas bentónicas, llegando a ingerir incluso otros equinodermos (Hendler et al. 1995, Ganmanee et al. 2003, Vala 2005, Caregnato et al. 2009). Los representantes de Astropecten han sido descritos como depredadores activos con una dieta generalista, aunque presentando preferencias por gastrópodos y bivalvos pequeños, siendo capaces de localizar presas enterradas y de excavar el sustrato para encontrarlas (Wells et al. 1961, Lera 1980, Bitter \& Penchaszadeh 1983, Penchaszadeh \& Lera 1983, Gammanee et al. 2003, Caregnato et al. 2009).

La ecorregión Delta del Orinoco es una de las trece en las que se ha dividido la zona marino-costera de Venezuela (Miloslavich et al. 2005). Los estudios realizados en esta región han sido escasos y discontinuos en el espacio y en el tiempo, por lo que existe una carencia de información sobre la distribución, ecología y biología de los organismos del área (Martín et al. 2007).

El objetivo de este trabajo fue determinar la distribución espacial y temporal, los parámetros poblacionales en base a la talla y peso de los individuos, y la dieta de $A$. marginatus en la ecorregión Delta del Orinoco, Venezuela.

\section{MATERIALES Y MÉTODOS}

Área de estudio: Comprendió la ecorregión Delta del Orinoco, la cual abarca las aguas del Océano Atlántico venezolano, frente a la desembocadura del Río Orinoco y el Golfo de Paria. Esta zona se caracteriza por tener fondos fangosos y arenosos, así como una alta carga de sedimentos y turbidez (Miloslavich \& Klein 2008). Se dividió el área de muestreo en tres sectores: Golfo de Paria, Boca de Serpiente y Plataforma Deltana. El material fue colectado durante los muestreos de megabentos de los proyectos Línea Base Ambiental Plataforma Deltana (LBAPD) y Corocoro Fase I (CFI), desarrollados por el Instituto de Tecnología y Ciencias Marinas-Universidad Simón Bolívar (INTECMAR-USB) para Petróleos de Venezuela, S.A. (PDVSA). El proyecto LBAPD fue realizado en dos campañas: una en la época de sequía (octubre 2004) y otra en la de lluvia (finales de mayo y principio de junio 2005), muestreándose 39 estaciones por campaña (con una muestra por estación) en toda la ecorregión: seis en Golfo de Paria, cuatro en Boca de Serpiente y 29 en Plataforma Deltana. Los muestreos del proyecto CFI fueron realizados en tres campañas sólo en el Golfo de Paria, dos durante la época de lluvia (junio y agosto 2006) y una en época de sequía (octubre 2006), habiéndose muestreado 18 estaciones por campaña y tres repeticiones por estación (Cuadro 1).

Recolección de las muestras: Las muestras de megabentos fueron recolectadas a profundidades entre $\operatorname{los} 8$ y $83 \mathrm{~m}$, utilizando una red de arrastre de $1.8 \mathrm{~m}$ de ancho por $0.5 \mathrm{~m}$ de alto y una abertura de malla de $1.0 \mathrm{~cm}$, la cual iba a bordo de un buque oceanográfico (Hermano Ginés (FLASA-EDIMAR) para las muestras del proyecto LBAPD y el Intrepido para las muestras del proyecto CFI). Para todos los muestreos, se tomaron las coordenadas de inicio y final del arrastre para calcular la distancia recorrida, así como el área del barrido. El tiempo durante el cual se realizaba el arrastre era dependiente de la consistencia del sedimento del fondo marino, por lo que los tiempos variaron entre los siete y doce minutos; durante los cuales se mantenía una velocidad cercana a los dos nudos (1 825m/h).

El megabentos fue separado en cada embarcación con un tamiz con abertura de malla de $1 \mathrm{~cm}$. Todas las muestras fueron colocadas en bolsas herméticamente selladas y 
CUADRO 1

Datos de las estaciones muestreadas en los proyectos LBAPD y CFI

TABLE 1

Data from sampling zones in LBAPD and CFI projects

\begin{tabular}{|c|c|c|c|c|c|c|c|c|c|}
\hline \multicolumn{7}{|c|}{ Proyecto LBAPD } & \multicolumn{3}{|c|}{ Proyecto CFI } \\
\hline $\begin{array}{l}\text { Estación } \\
\text { en este } \\
\text { trabajo }\end{array}$ & $\begin{array}{c}\text { Estación } \\
\text { original }\end{array}$ & $\begin{array}{l}\text { Coordenadas } \\
\text { Longitud }\end{array}$ & $\begin{array}{c}\text { s geográficas } \\
\text { Latitud }\end{array}$ & $\begin{array}{l}\text { Profundidad } \\
\quad \text { (m) }\end{array}$ & $\begin{array}{c}\text { Estación } \\
\text { en este } \\
\text { trabajo }\end{array}$ & $\begin{array}{l}\text { Estación } \\
\text { original }\end{array}$ & $\begin{array}{l}\text { Coordenadas } \\
\text { Longitud }\end{array}$ & $\begin{array}{l}\text { geográficas } \\
\text { Latitud }\end{array}$ & $\begin{array}{l}\text { Profundidad } \\
\quad \text { (m) }\end{array}$ \\
\hline 1 & 1 & $-62^{\circ} 47.251^{\prime}$ & $10^{\circ} 32.659^{\prime}$ & 9.0 & 1 & RN1S24 & $-62^{\circ} 13.011^{\prime}$ & $10^{\circ} 18.518^{\prime}$ & 32.0 \\
\hline 2 & 2 & $-62^{\circ} 31.123^{\prime}$ & $10^{\circ} 34.689^{\prime}$ & 14.5 & 2 & RN1S1 & $-62^{\circ} 12.040^{\prime}$ & $10^{\circ} 18.794^{\prime}$ & 29.0 \\
\hline 3 & 3 & $-62^{\circ} 15.207^{\prime}$ & $10^{\circ} 37.462$ & 28.5 & 3 & RN1S17 & $-62^{\circ} 12.484^{\prime}$ & $10^{\circ} 17.876^{\prime}$ & 31.0 \\
\hline 4 & 4 & $-62^{\circ} 35.695^{\prime}$ & $10^{\circ} 23.211^{\prime}$ & 10.5 & 4 & I1S7 & $-62^{\circ} 08.921^{\prime}$ & $10^{\circ} 15.544^{\prime}$ & 29.0 \\
\hline 5 & 5 & $-62^{\circ} 16.973^{\prime}$ & $10^{\circ} 25.918^{\prime}$ & 25.9 & 5 & I1S2 & $-62^{\circ} 08.815^{\prime}$ & $10^{\circ} 15.114^{\prime}$ & 30.0 \\
\hline 6 & 6 & $-62^{\circ} 18.942^{\prime}$ & $10^{\circ} 09.538^{\prime}$ & 10.5 & 6 & I1S8 & $-62^{\circ} 08.990^{\prime}$ & $10^{\circ} 14.628^{\prime}$ & 29.0 \\
\hline 7 & 7 & $-62^{\circ} 00.220^{\prime}$ & $10^{\circ} 01.530^{\prime}$ & 36.5 & 7 & RS1S9 & $-62^{\circ} 05.097^{\prime}$ & $10^{\circ} 12.654^{\prime}$ & 29.0 \\
\hline 8 & 8 & $-61^{\circ} 80.379^{\prime}$ & $09^{\circ} 96.879^{\prime}$ & 31.2 & 8 & RS1S3 & $-62^{\circ} 04.983^{\prime}$ & $10^{\circ} 12.077^{\prime}$ & 25.0 \\
\hline 9 & 10 & $-61^{\circ} 65.427^{\prime}$ & $09^{\circ} 96.893^{\prime}$ & 24.0 & 9 & RS1S10 & $-62^{\circ} 04.968^{\prime}$ & $10^{\circ} 11.606^{\prime}$ & 28.0 \\
\hline 10 & 12 & $-61^{\circ} 50.219^{\prime}$ & $09^{\circ} 94.971^{\prime}$ & 27.0 & 10 & RN2S15 & $-62^{\circ} 15.997^{\prime}$ & $10^{\circ} 13.107^{\prime}$ & 16.0 \\
\hline 11 & 14 & $-61^{\circ} 19.297^{\prime}$ & $09^{\circ} 80.356^{\prime}$ & 22.0 & 11 & RN2S5 & $-62^{\circ} 16.213^{\prime}$ & $10^{\circ} 12.747^{\prime}$ & 14.0 \\
\hline 12 & 17 & $-61^{\circ} 01.666^{\prime}$ & $09^{\circ} 84.098^{\prime}$ & 46.5 & 12 & RN2S16 & $-62^{\circ} 16.098^{\prime}$ & $10^{\circ} 12.289^{\prime}$ & 10.0 \\
\hline 13 & 18 & $-60^{\circ} 95.721^{\prime}$ & $09^{\circ} 71.767^{\prime}$ & 20.5 & 13 & $\mathrm{I} 2 \mathrm{~S} 13$ & $-62^{\circ} 11.975^{\prime}$ & $10^{\circ} 09.956^{\prime}$ & 17.0 \\
\hline 14 & 19 & $-60^{\circ} 92.464^{\prime}$ & $09^{\circ} 65.010^{\prime}$ & 8.0 & 14 & $\mathrm{I} 2 \mathrm{~S} 4$ & $-62^{\circ} 12.545^{\prime}$ & $10^{\circ} 09.793^{\prime}$ & 10.0 \\
\hline 15 & 23 & $-60^{\circ} 82.713^{\prime}$ & $09^{\circ} 74.727^{\prime}$ & 41.0 & 15 & $\mathrm{I} 2 \mathrm{~S} 14$ & $-62^{\circ} 12.769^{\prime}$ & $10^{\circ} 09.336^{\prime}$ & 14.0 \\
\hline 16 & 24 & $-60^{\circ} 79.254^{\prime}$ & $09^{\circ} 67.895^{\prime}$ & 35.5 & 16 & $\mathrm{RS} 2 \mathrm{~S} 11$ & $-62^{\circ} 08.775^{\prime}$ & $10^{\circ} 08.573^{\prime}$ & 21.0 \\
\hline 17 & 25 & $-60^{\circ} 73.199^{\prime}$ & $09^{\circ} 55.925^{\prime}$ & 17.5 & 17 & RS2S6 & $-62^{\circ} 09.040^{\prime}$ & $10^{\circ} 08.108^{\prime}$ & 24.0 \\
\hline 18 & 26 & $-60^{\circ} 36.227^{\prime}$ & $09^{\circ} 24.315^{\prime}$ & 12.0 & 18 & $\mathrm{RS} 2 \mathrm{~S} 12$ & $-62^{\circ} 09.536^{\prime}$ & $10^{\circ} 7.855^{\prime}$ & 19.0 \\
\hline 19 & 27 & $-60^{\circ} 29.000^{\prime}$ & $09^{\circ} 14.900^{\prime}$ & 9.0 & & & & & \\
\hline 20 & 28 & $-60^{\circ} 63.560^{\prime}$ & $09^{\circ} 79.086^{\prime}$ & 58.0 & & & & & \\
\hline 21 & 29 & $-60^{\circ} 60.029^{\prime}$ & $09^{\circ} 72.091^{\prime}$ & 53.0 & & & & & \\
\hline 22 & 30 & $-60^{\circ} 53.751^{\prime}$ & $09^{\circ} 59.626^{\prime}$ & 52.0 & & & & & \\
\hline 23 & 31 & $-60^{\circ} 45.645^{\prime}$ & $09^{\circ} 43.591^{\prime}$ & 52.5 & & & & & \\
\hline 24 & 32 & $-60^{\circ} 37.365^{\prime}$ & $09^{\circ} 27.120^{\prime}$ & 41.0 & & & & & \\
\hline 25 & 33 & $-60^{\circ} 42.640^{\prime}$ & $09^{\circ} 83.842^{\prime}$ & 63.0 & & & & & \\
\hline 26 & 34 & $-60^{\circ} 39.113$ & $09^{\circ} 76.664^{\prime}$ & 62.0 & & & & & \\
\hline 27 & 35 & $-60^{\circ} 32.702^{\prime}$ & $09^{\circ} 63.624^{\prime}$ & 62.0 & & & & & \\
\hline 28 & 36 & $-60^{\circ} 24.945^{\prime}$ & $09^{\circ} 47.862^{\prime}$ & 60.0 & & & & & \\
\hline 29 & 37 & $-60^{\circ} 16.710^{\prime}$ & $09^{\circ} 31.093^{\prime}$ & 60.0 & & & & & \\
\hline 30 & 38 & $-60^{\circ} 28.691^{\prime}$ & $09^{\circ} 87.014^{\prime}$ & 74.0 & & & & & \\
\hline 31 & 39 & $-60^{\circ} 25.086^{\prime}$ & $09^{\circ} 79.728^{\prime}$ & 73.0 & & & & & \\
\hline 32 & 40 & $-60^{\circ} 18.450^{\prime}$ & $09^{\circ} 66.328^{\prime}$ & 70.0 & & & & & \\
\hline 33 & 41 & $-60^{\circ} 10.751^{\prime}$ & $09^{\circ} 50.783^{\prime}$ & 71.0 & & & & & \\
\hline 34 & 42 & $-60^{\circ} 02.365^{\prime}$ & $09^{\circ} 33.847^{\prime}$ & 66.0 & & & & & \\
\hline 35 & 43 & $-60^{\circ} 13.219^{\prime}$ & $09^{\circ} 90.543^{\prime}$ & 81.0 & & & & & \\
\hline 36 & 44 & $-60^{\circ} 09.647^{\prime}$ & $09^{\circ} 83.101^{\prime}$ & 81.0 & & & & & \\
\hline 37 & 45 & $-60^{\circ} 03.002^{\prime}$ & $09^{\circ} 69.270^{\prime}$ & 83.0 & & & & & \\
\hline 38 & 46 & $-59^{\circ} 95.631^{\prime}$ & $09^{\circ} 53.917^{\prime}$ & 83.0 & & & & & \\
\hline 39 & 47 & $-59^{\circ} 87.359^{\prime}$ & $09^{\circ} 36.720^{\prime}$ & 77.0 & & & & & \\
\hline
\end{tabular}


preservadas con una solución de formalina diluida con agua de mar al 10\%, así como debidamente etiquetadas y catalogadas. Una vez en el laboratorio, las muestras fueron tamizadas nuevamente usando tamices de $5 \mathrm{~mm}$ de abertura de malla y luego separadas, determinadas y preservadas con alcohol al 70\%. Posteriormente, se tomaron sólo los ejemplares de la estrella A. marginatus para la realización de este trabajo. Algunos de dichos ejemplares fueron depositados en la colección de equinodermos del Museo de Ciencias Biológicas de la Universidad Simón Bolívar, Caracas, Venezuela.

Procesamiento de las muestras: Se midió con un vernier $( \pm 0.001 \mathrm{~cm})$ la talla $(\mathrm{mm})$ de los ejemplares de A. marginatus tomada desde el centro de la estrella hasta el extremo de un brazo completo (R) (Fig. 1) y posteriormente, se determinó el peso húmedo de los mismos $( \pm 0.1 \mathrm{gr})$. El contenido estomacal fue extraído cortando cuidadosamente la parte oral de la estrella, incluyendo la región de la boca y los brazos. El estómago se colocó en una cápsula de Petri y con la ayuda de pinzas finas y una lupa estereoscópica se separó los componentes alimenticios de las partes del estómago. Los componentes de la dieta fueron determinados, contados y en algunos casos medidos (por ejemplo algunos crustáceos, moluscos, escamas de peces y holoturoideos).

Para determinar la estructura de la población se realizaron correlaciones entre las tallas y pesos húmedos de los organismos mediante gráficas de dispersión de talla vs peso y se determinaron las densidades (ind $/ \mathrm{m}^{2}$ ) y la biomasa $\left(\mathrm{mg} / \mathrm{m}^{2}\right)$ de individuos en cada localidad para las fechas de muestreo. Las diferencias poblacionales significativas entre los parámetros de densidad y biomasa fueron analizadas utilizando pruebas Chi-cuadrado $\left(\chi^{2}\right)$ por periodo en cada sector y entre sectores. Las clases de talla de los individuos fueron calculadas mediante la regla de Sturges (1926).

El número de presas consumidas y de categorías alimenticias encontradas en cada estómago y el tamaño de la estrella se relacionaron mediante gráficos de dispersión. Para medir la diversidad de la dieta se utilizó el índice de Shannon $\left(\mathrm{H}^{\prime}\right)$, considerándose solamente aquellas categorías cuantificables en individuos discretos. Como este índice varía en un intervalo entre 0 e infinito, se estandarizó entre 0-1 mediante el índice de equidad de Pielou J' con la fórmula $\mathrm{J}^{\prime}=\mathrm{H}^{\prime} / \log _{2} \mathrm{n}$, donde $\mathrm{H}^{\prime}$ es el valor del índice de Shannon y n el número total de posibles categorías alimenticias (Krebs 1998).

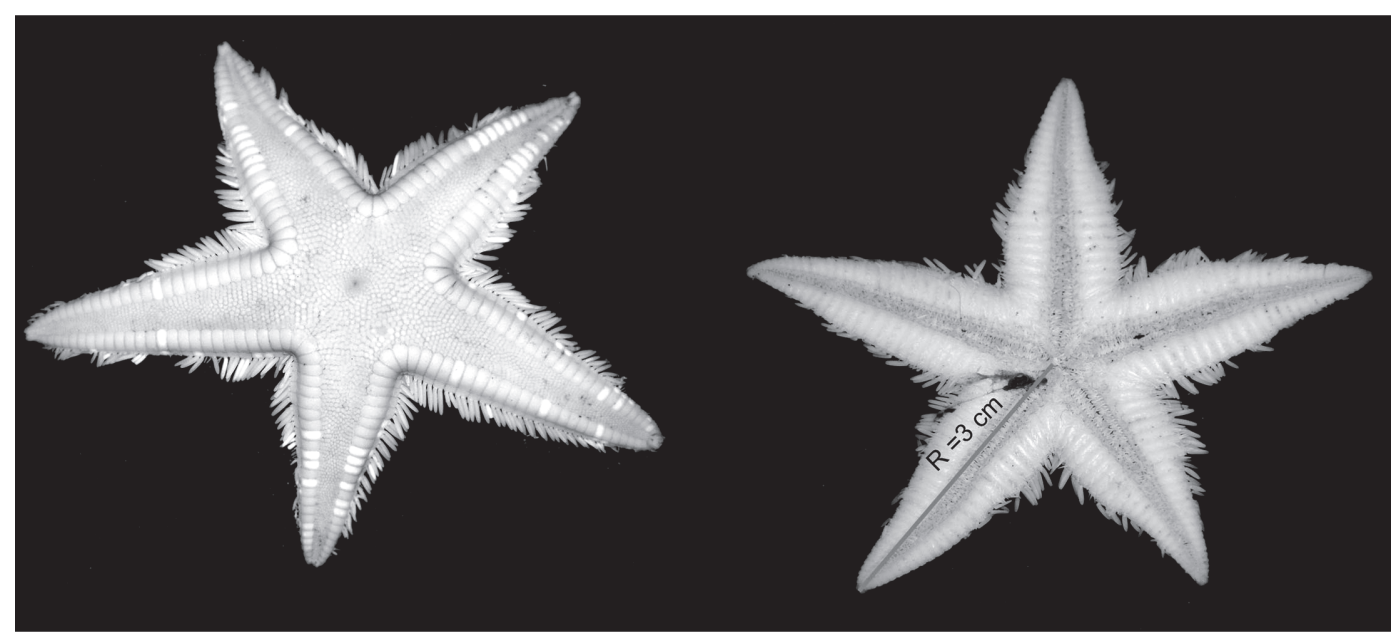

Fig. 1. Ejemplar de Astropecten marginatus con la medida de radio del brazo (R) tomada.

Fig. 1. Specimen of Astropecten marginatus with arm radio (R) measured. 
La frecuencia numérica (FN) representó el número total de individuos de cada categoría alimenticia referido al total de individuos encontrados en el contenido estomacal. La frecuencia de aparición (FA), representó el número de estómagos donde apareció un ítem alimenticio determinado entre el número total de estómagos con alimento, expresado en porcentaje. Se realizaron pruebas de $\chi^{2}$ a las diferentes frecuencias de tallas para comparar su variación en el tiempo y entre las localidades muestreadas. Finalmente, se estimó el grado de semejanza entre los contenidos estomacales de los organismos usando el índice de Bray-Curtis y a partir de la matriz de similitud se efectuó un análisis de ordenación con la técnica de escalamiento multidimensional no métrico (nMDS; Clarke \& Gorley 2006). Para confirmar si los contenidos estomacales mostraban algún arreglo discernible y estadísticamente significativo se consideró el nivel de stress del nMDS y se aplicó el análisis de similitud (SIMPER; Clarke \& Gorley 2006). Esta prueba es análoga a un análisis de varianza no paramétrico de una vía, y se basa en el re-muestreo aleatorio de los valores de similitud entre sitios.

\section{RESULTADOS}

Distribución: Se determinaron un total de 570 ejemplares de A. marginatus provenientes de las muestras recolectadas en el periodo 2004-2005: 250 en la época de sequía y 320 en la época de lluvia. Para los tres sectores, se encontró mayor cantidad de estaciones con estrellas en la época de sequía, siendo mayor el porcentaje de aparición en el sector Golfo de Paria. El total de individuos provenientes de los muestreos del 2006 fue de 306 estrellas: 187 en época de lluvia y 119 en época de sequía (Cuadro 2).

De manera general, se encontraron estrellas entre $8-83 \mathrm{~m}$, siendo el intervalo de aparición más frecuente entre 9-40m. En el Golfo de Paria se obtuvieron individuos entre 9-24m, con mayor abundancia entre $9-18.4 \mathrm{~m}$. En el sector Boca de Serpiente solamente a los $36.5 \mathrm{~m}$, mientras que en el sector Plataforma

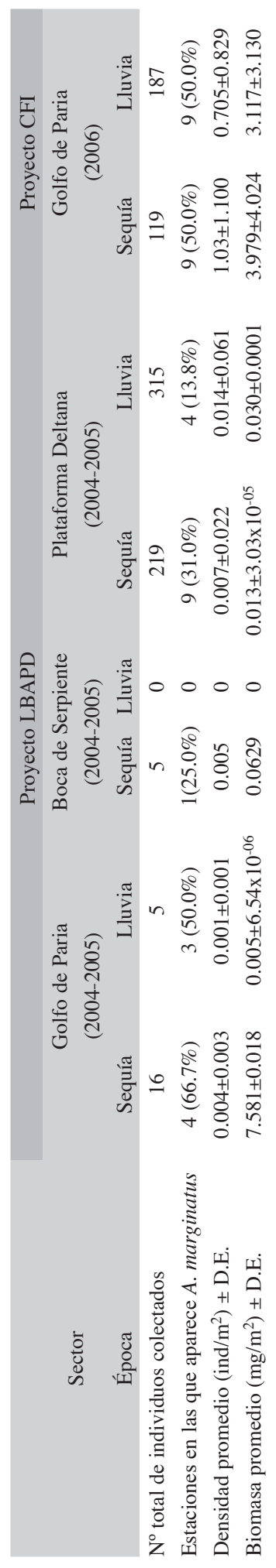


Deltana el intervalo de profundidad varió entre 8-83m, siendo mayor la abundancia de estrellas a los $17 \mathrm{~m}$ en la época de sequía y a los $43 \mathrm{~m}$ en la época de lluvia. La distribución del promedio de tallas por profundidad no mostró ningún patrón en los sectores muestreados, no habiéndose observado tampoco una relación entre el tamaño promedio de la estrella y la profundidad a la que fue colectada (Fig. 2).

Parámetros Poblacionales: La tendencia general en los tres sectores fue una disminución de la densidad promedio de las estrellas en la época de lluvia, tanto en el periodo 2004-2005 como en el 2006; sin embargo, al tomar los datos promedio del sector Plataforma Deltana, los valores de densidad indican lo contrario, esto debido a una sola estación, en la cual se encontró un número de individuos equivalente a casi la mitad de las estrellas identificadas en el proyecto LBAPD (2004-2005) (Cuadro 2). Los sectores con mayor densidad correspondieron al Golfo de Paria en el 2006 y a Plataforma Deltana durante 2004-2005 sin embargo, se
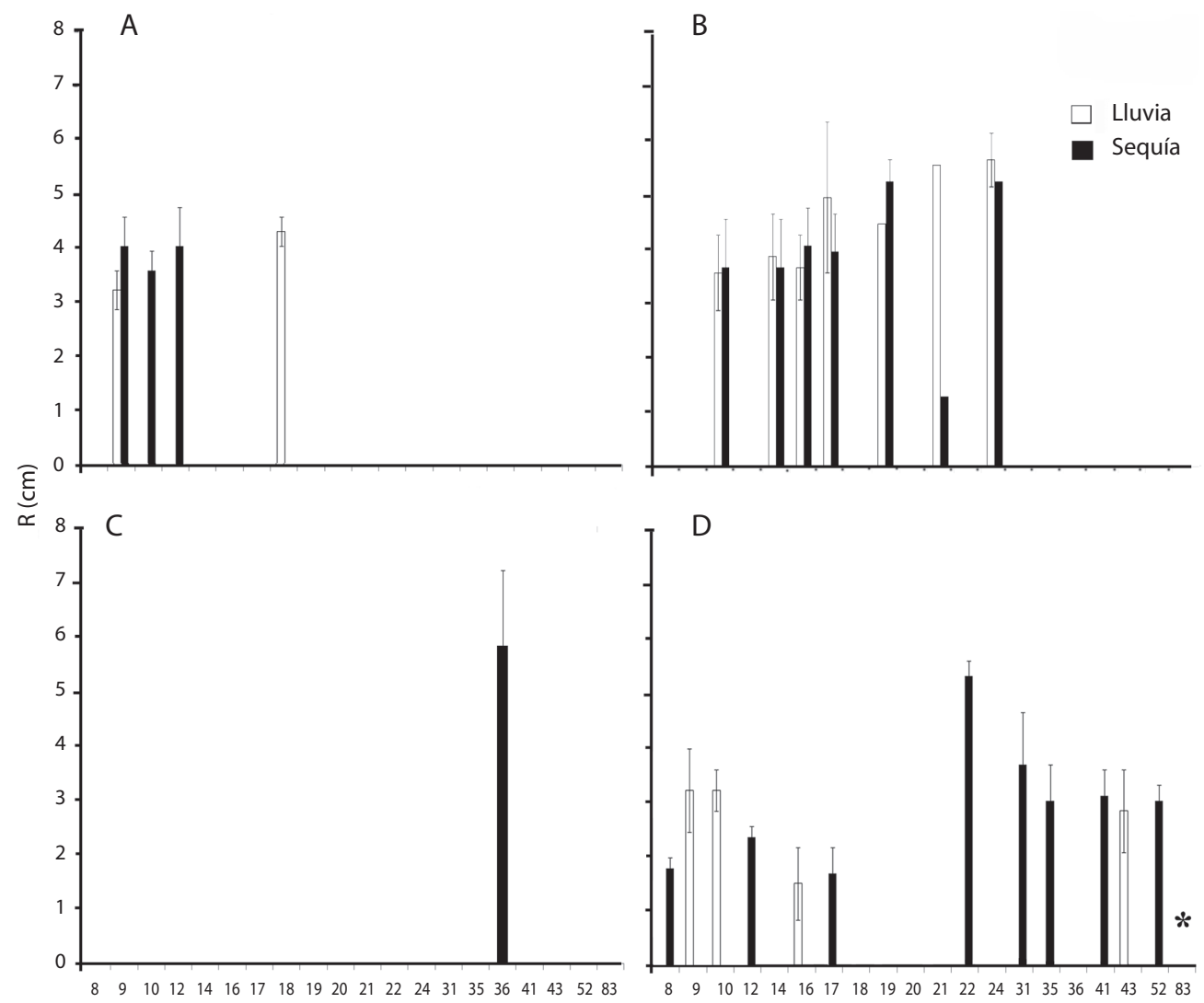

Profundidad (m)

Fig. 2. Radio (R) promedio (cm) del brazo de A. marginatus por cada profundidad a la que fue encontrada en el sector: (A) Golfo de Paria (2004-2005) LBAPD, (B) Golfo de Paria (2006) CFI, (C) Boca de Serpiente (2004-2005) LBAPD, (D) Plataforma Deltana (2004-2005) LBAPD. Las líneas sobre las barras indican la desviación estándar de los valores. * Los ejemplares aquí encontrados no presentaban ningún brazo completo.

Fig. 2. Average of arm radio (R) from A. marginatus in each found deep in the sections of: (A) Golfo de Paria (2004-2005) LBAPD, (B) Golfo de Paria (2006) CFI, (C) Boca de Serpiente (2004-2005) LBAPD, (D) Plataforma Deltana (2004-2005) LBAPD. The row above the columns means the standard deviation of the values. * Sampled individuals didn't have any full arm. 
presenta una elevada desviación estándar en los valores de densidad de este último sector (Cuadro 2). No se observaron diferencias significativas en la distribución de densidades entre la época de lluvia o sequía, utilizando los datos de toda la zona de estudio. Sin embargo, cuando se evaluó cada sector por separado, sí existían diferencias tanto en el Golfo de Paria como en la Plataforma Deltana $\left(\chi^{2}, \mathrm{p}<0.05\right)$ (Cuadro 3).

La biomasa también presentó una disminución en la época de lluvia, con excepción del sector Plataforma Deltana (2004-2005), donde se incrementó en la época de lluvia a causa de la estación con mayor densidad, la tendencia general del resto de las estaciones coincide con lo que ocurre en el Golfo de Paria (Cuadro 2), siendo éste el que presentó la mayor biomasa. No se observaron diferencias significativas en la distribución de biomasa entre las épocas en toda la zona de estudio $\left(\chi^{2}, \mathrm{p}<0.05\right)$, sin embargo, al analizar por sector, tanto en el Golfo de Paria como en la Plataforma Deltana en el periodo 2004-2005 se encontraron diferencias significativas, pero no para el Golfo de Paria en el $2006\left(\chi^{2}, \mathrm{p}<0.05\right)$ (Cuadro 3).

El intervalo de tallas medidas varió entre $0.7-7.8 \mathrm{~cm}$. Se observó que independientemente del sector, época o año de muestreo, a medida que aumenta el radio $(\mathrm{R})$ hay un incremento en el peso (Fig. 3).

Con base en las dimensiones de $\mathrm{R}$, en el sector Golfo de Paria, independientemente de la época y año evaluado, el intervalo de talla estuvo entre $3.1-5.4 \mathrm{~cm}$, siendo más frecuente el intervalo de entre $3.9-4.6 \mathrm{~cm}$. La baja cantidad de individuos encontrados en el sector Boca de Serpiente no permitió ver una tendencia en la distribución de las clases de talla. En el sector Plataforma Deltana, se encontró que la abundancia de individuos por clases de tallas varió según la época, encontrándose estrellas de menor tamaño en época de sequía y estrellas de mayor tamaño en época de lluvia. El intervalo más frecuente fue entre $1.5-2.2 \mathrm{~cm}$ en el 2004 y entre $3.1-3.8 \mathrm{~cm}$ en el 2005 (Cuadro 4). No se encontraron diferencias significativas en la distribución de clases de talla entre la época de lluvia y sequía en toda la zona de estudio, salvo para el sector Plataforma Deltana $\left(\chi^{2}, \mathrm{p}<0.05\right)$ (Cuadro 3).

Dieta: En las estrellas analizadas, provenientes del sector Golfo de Paria, se encontró durante 2004-2005 un total de 249 presas consumidas, agrupadas en 19 categorías alimenticias en época de sequía y 13 en época de lluvia. En los muestreos del 2006, se encontró un total

CUADRO 3

Valores obtenidos de chi-cuadrado en la distribución de los diferentes parámetros poblacionales en los sectores de estudio y en toda la ecorregión entre las épocas de lluvia y sequía

TABLE 3

Values obtained of chi-square on the population parameters distribution in the studied sections and the whole ecoregion between rainy and dry seasons

\begin{tabular}{lcccccc} 
& Proyecto CFI & \multicolumn{3}{c}{ Proyecto LBAPD } & \multicolumn{2}{c}{ Ecorregión Delta del Orinoco } \\
& $\begin{array}{c}\text { Golfo de Paria } \\
(2006)\end{array}$ & $\begin{array}{c}\text { Golfo de Paria } \\
(2004-2005)\end{array}$ & $\begin{array}{c}\text { Boca de Serpiente } \\
(2004-2005)\end{array}$ & $\begin{array}{c}\text { Plataforma Deltana } \\
(2004-2005)\end{array}$ & (lluvia) & (sequía) \\
Biomasa & 18.43 & $6.95 \times 10^{-05}$ & - & 0.00044 & 54.66 & $7.4 \times 10^{8}$ \\
Densidad & 3.45 & 0.00039 & - & 0.29 & 25.39 & 37.00 \\
Tallas & 118.50 & 528.77 & 560.04 & 1.17 & 696.38 & 911.01
\end{tabular}

p-valor $<0.05,1$ grado de libertad. (*) valores significativos. p-value $<0.05,1$ freedom degree. $(*)$ significative values. 


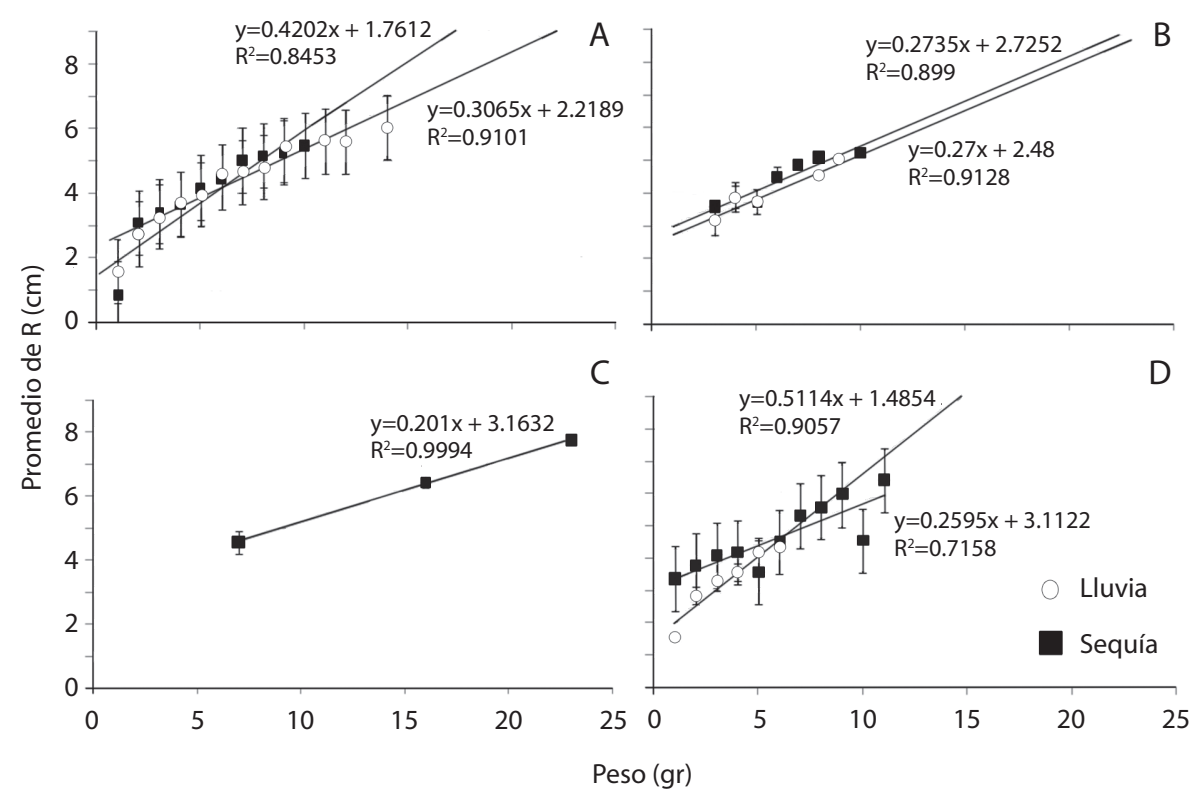

Fig. 3. Regresión lineal entre la talla de A. marginatus y su peso húmedo en las diferentes épocas muestreadas en los sectores: (A) Golfo de Paria (2004-2005) LBAPD, (B) Golfo de Paria (2006) CFI, (C) Boca de Serpiente (2004-2005) LBAPD, (D) Plataforma Deltana (2004-2005) LBAPD.

Fig. 3. Linear regression between the size and weight of A. marginatus in different times in the sections of: (A) Golfo de Paria (2004-2005) LBAPD, (B) Golfo de Paria (2006) CFI, (C) Boca de Serpiente (2004-2005) LBAPD, (D) Plataforma Deltana (2004-2005) LBAPD.

CUADRO 4

Número de individuos recolectados en cada sector por cada clase de talla evaluada

TABLE 4

Number of individuals for each size class found in every section

\begin{tabular}{|c|c|c|c|c|c|c|c|c|}
\hline \multirow{2}{*}{$\begin{array}{l}\text { Clase de talla } \\
\text { (cm) }\end{array}$} & \multicolumn{2}{|c|}{$\begin{array}{c}\text { Golfo de Paria } \\
\text { (2004-2005) }\end{array}$} & \multicolumn{2}{|c|}{$\begin{array}{l}\text { Boca de Serpiente } \\
\quad(2004-2005)\end{array}$} & \multicolumn{2}{|c|}{$\begin{array}{l}\text { Plataforma Deltana } \\
\quad(2004-2005)\end{array}$} & \multicolumn{2}{|c|}{$\begin{array}{l}\text { Golfo de Paria } \\
\text { (2006) }\end{array}$} \\
\hline & Sequía & Lluvia & Sequía & Lluvia & Sequía & Lluvia & Sequía & Lluvia \\
\hline $0.7-1.4$ & & & & & 25 & 43 & 5 & 2 \\
\hline $1.5-2.2$ & & & & & 88 & 59 & & 3 \\
\hline $2.3-3.0$ & & & & & 44 & 54 & 5 & 19 \\
\hline $3.1-3.8$ & 4 & 2 & & & 41 & 118 & 54 & 76 \\
\hline $3.9-4.6$ & 8 & 2 & 1 & & 21 & 40 & 36 & 60 \\
\hline $4.7-5.4$ & 4 & 1 & 1 & & 5 & 1 & 17 & 18 \\
\hline $5.5-6.2$ & & & & & 2 & & 2 & 8 \\
\hline $6.3-7.0$ & & & 2 & & & & & \\
\hline $7.1-7.8$ & & & 1 & & & & & 1 \\
\hline
\end{tabular}


de 751 presas consumidas, agrupadas en 22 categorías alimenticias en época de lluvia y 19 en época de sequía. En el sector Boca de Serpiente, se encontraron un total de 110 presas consumidas, agrupadas en cinco categorías alimenticias. En el sector Plataforma Deltana se encontró un total de 1866 presas consumidas, agrupadas en 32 grandes categorías alimenticias en época de sequía y 28 en época de lluvia (Fig. 4).
Las principales categorías alimenticias encontradas fueron: escafópodos, bivalvos, gasterópodos, decápodos, ostrácodos, peracáridos, picnogónidos, poliquetos, restos calcáreos de asteroideos $\mathrm{u}$ ofiuroideos $\mathrm{y}$ escamas de peces, además de algas, fragmentos vegetales y sedimento, y el material restante fue agrupado dentro de la categoría "otros" por presentar frecuencias de aparición menores al 15\% (Fig. 4).

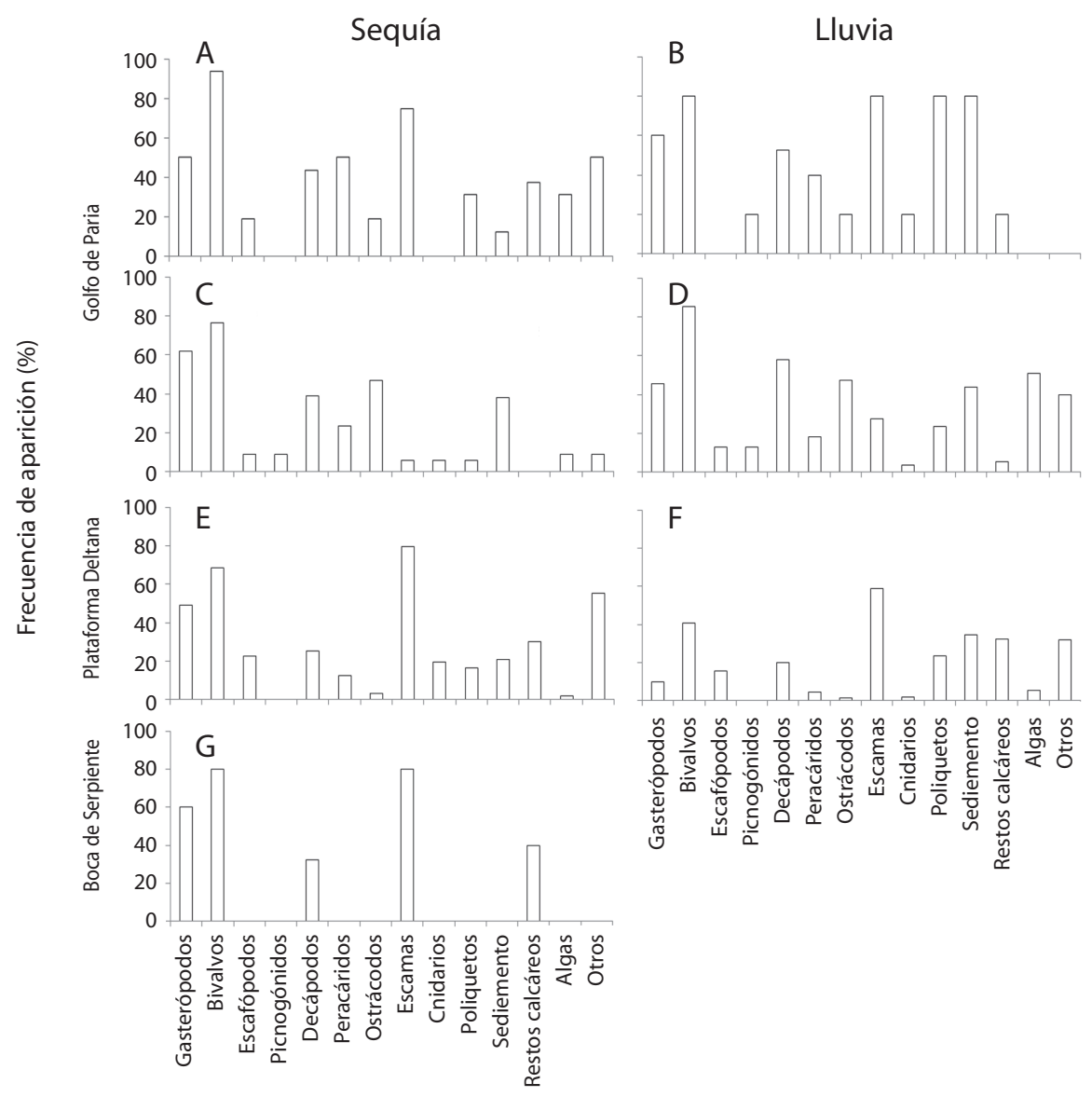

Fig. 4. Frecuencia de aparición (\%) de las principales categorías alimenticias en el sector Golfo de Paria (A) en época de sequía (2004) LBAPD; (B) época de lluvia (2005) LBAPD; (C) época de sequía (2006) CFI; (D) época de lluvia (2006) CFI; (E) en el sector Plataforma Delta época de sequía (2004) LBAPD; (F) en el sector Plataforma Deltana época de lluvia (2005) LBAPD; (G) en el sector Boca de Serpiente época de sequía (2004) LBAPD.

Fig. 4. Appearance frequency (\%) of principals food items in: the Golfo de Paria section in (A) dry season (2004) LBAPD; (B) raining season (2005) LBAPD; (C) dry season (2006) LBAPD; (D) raining season (2006) LBAPD; (E) dry season in the Plataforma Deltana section (2004) LBAPD; (F) raining season in the Plataforma Deltana section (2005) LBAPD; (G) dry season in the Boca de Serpiente section (2004) LBAPD. 
No se observó patrón de variación alguno en los componentes de la dieta respecto a la talla del individuo, independientemente de la época, sector o año del muestreo. En cuanto al tamaño de las presas consumidas, éstas por lo general son de tallas pequeñas, pudiendo variar entre menores a $1.0 \mathrm{~mm}$ (algunos crustáceos) hasta $17.0 \mathrm{~mm}$ (holotúrido), constituyendo este límite, un tamaño superior al del diámetro del disco central de la estrella donde apareció.

Al calcular el índice de Shannon (H'), con el fin de verificar la diversidad de la dieta se obtuvieron valores muy cercanos a 3.0 tanto en el Golfo de Paria como en el sector Plataforma Deltana en las dos épocas del año y los dos periodos muestreados, lo que refleja un hábito alimenticio generalista. Para el sector Boca de Serpiente, la diversidad fue menor $\left(H^{\prime}=0.9\right)$, posiblemente por la baja densidad de estrellas que se encontraron. Al calcular el índice de equidad de Pielou los valores fueron cercanos a 1.0, con excepción del sector Boca de Serpiente (Cuadro 5), por lo que se observa que los componentes de la dieta de esta especie son igualmente abundantes.

$\mathrm{La}$ mayor frecuencia numérica $(\mathrm{FN}) \mathrm{y}$ de aparición de presas (FA) encontrada en el contenido estomacal de las estrellas independientemente de la época del año o sector correspondió a los bivalvos (FA superiores al $50 \%$ en todos los casos, llegando a ocupar una frecuencia de $93.8 \%$ en época de sequía durante los muestreos de 2004-2005; FN superior a $30 \%$ ), seguidos por los gasterópodos (FA superiores al 30\% y FN superior al 13\%). Las escamas de peces, poliquetos y el sedimento también ocuparon frecuencias de aparición mayores al 30\% y los ostrácodos frecuencias numéricas superiores al 15\% (Fig. 4). Los ostrácodos en particular presentaron un notable aumento en la época de sequía en el Golfo de Paria durante el 2006, pasando a ser en esta última época el elemento más abundante en la dieta de $A$. marginatus en el sector.

Al agrupar los valores obtenidos por el total de estrellas sin distinción de sector, tallas o épocas, se observa que la dieta de A. marginatus está conformada principalmente por moluscos $(47.4 \%)$, en especial bivalvos y gasterópodos $(\mathrm{FA}=56.3 \%$ y $35.0 \%$ respectivamente y $\mathrm{FN}=28.5 \%$ y $13.6 \%$ respectivamente), pudiendo ingerir además un gran número de componentes animales, vegetales e incluso no orgánicos como pequeñas piedras y sedimento, aunque estos últimos lo más probable es que sea de manera accidental (Fig. 5).

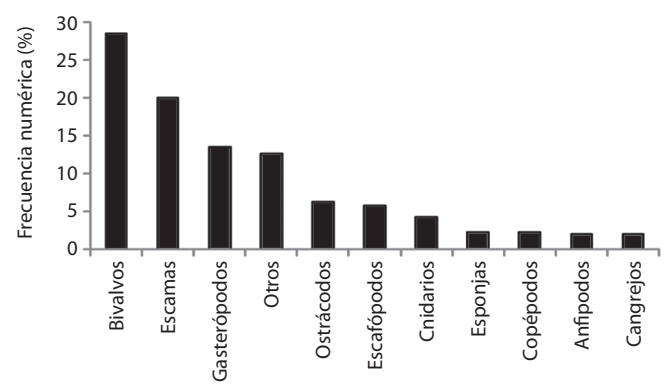

Fig. 5. Frecuencia numérica total de las principales categorías alimenticias encontradas en el contenido estomacal de A. marginatus.

Fig. 5. Total numeric frequency of the main food categories found in the stomach content of A. marginatus.

CUADRO 5

Índice de Shannon ( $\left.H^{\prime}\right)$ e índice de equidad de Pielou ( $\left.J^{\prime}\right)$ para ver la diversidad de la dieta de A. marginatus en los sectores estudiados

TABLE 5

Shannon $\left(H^{\prime}\right)$ and Pielou ( $\left.J^{\prime}\right)$ equity index to show the diet diversity of A. marginatus in the sections studied

\begin{tabular}{|c|c|c|c|c|c|c|c|c|}
\hline & \multicolumn{2}{|c|}{$\begin{array}{c}\text { Golfo de Paria } \\
\text { (2006) }\end{array}$} & \multicolumn{2}{|c|}{$\begin{array}{c}\text { Golfo de Paria } \\
(2004-2005)\end{array}$} & \multicolumn{2}{|c|}{$\begin{array}{c}\text { Boca de Serpiente } \\
(2004-2005)\end{array}$} & \multicolumn{2}{|c|}{$\begin{array}{c}\text { Plataforma Deltana } \\
\text { (2004-2005) }\end{array}$} \\
\hline & Sequía & Lluvia & Sequía & Lluvia & Sequía & Lluvia & Sequía & Lluvia \\
\hline $\mathrm{H}^{\prime}$ & 2.50 & 3.26 & 2.80 & 3.09 & 0.93 & & 3.12 & 3.10 \\
\hline J' & 0.64 & 0.77 & 0.74 & 0.89 & 0.46 & & 0.64 & 0.68 \\
\hline
\end{tabular}


Al comparar la dieta de las estrellas mediante un análisis de ordenación de escalamiento multidimensional no métrico (nMDS), en cuanto a los parámetros: época (lluvia y sequía), sector (Golfo de Paria, Boca de Serpiente y Plataforma Deltana), proyecto (CFI y LBAPD), clase de talla, y la combinación de estos cuatro parámetros por pareja (épocasector, época-proyecto, época-clase de talla, sector-proyecto, sector-clase de talla, proyectoclase de talla), no se observaron patrones de agrupación entre todos los parámetros evaluados con un valor de stress de 0.01 (Fig. 6). Al realizar los análisis de similitud (SIMPER) los

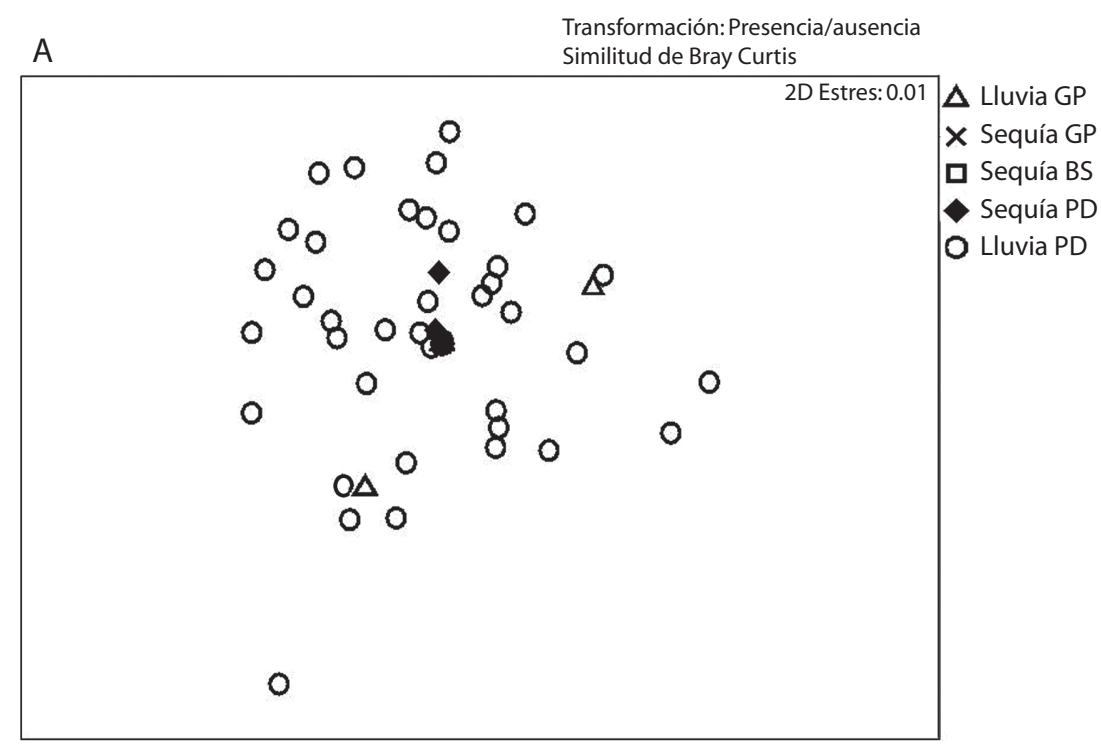

B

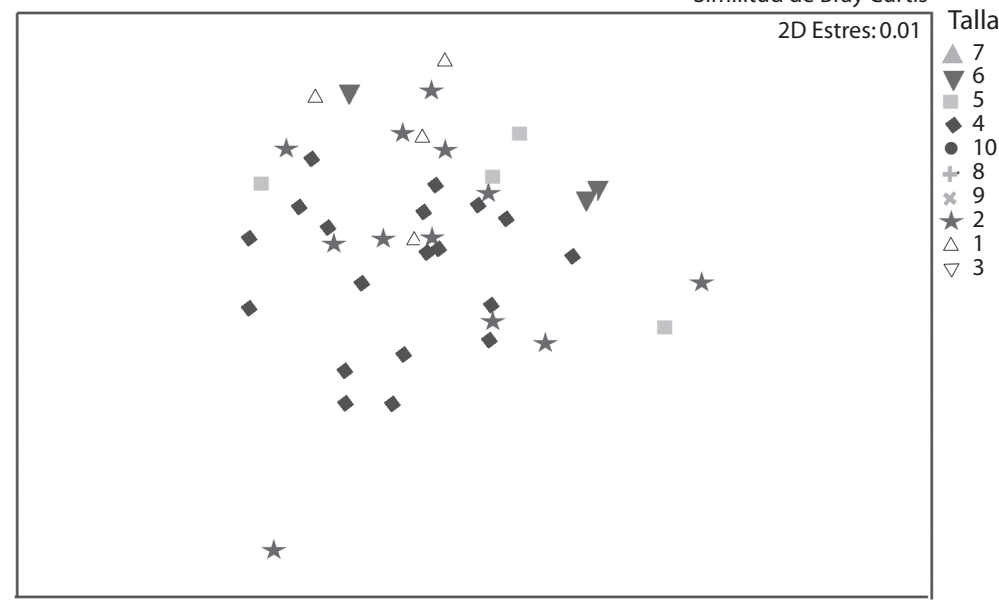

Fig. 6. Ejemplo de la ordenación de dos nMDS de los elementos alimenticios encontrados en la dieta de A. marginatus de la ecorregión Delta del Orinoco (A) comparación por época del año y sector (B) comparación por clases de talla.

Fig. 6. Example of two nMDS of the food items found in the A. marginatus diet at Orinoco Delta ecoregion (A) comparison by season and section $(\mathbf{B})$ comparison by size class. 
resultados mostraron que dentro de cada parámetro había muy baja similitud en la dieta, siendo en todos los casos menor al 40\% (Cuadro 6); y al comparar la dieta entre la combinación de los parámetros estudiados, se observó una alta disimilitud de la dieta, con valores superiores al $80 \%$ (Cuadro 7). Estos bajos valores de similitud en cada parámetro, así como entre los pares de parámetros comparados, se deben principalmente al grupo de los bivalvos, los cuales son uno de los más abundantes, evidenciándose la gran heterogeneidad de la dieta de esta especie, a pesar de la predominancia de los bivalvos como principal elemento alimenticio.

\section{DISCUSIÓN}

Distribución: Se infiere que la estrella A. marginatus presenta una distribución por parches en la ecorregión Delta del Orinoco, dada la baja presencia de la misma en el número total de estaciones muestreadas. Klein \& Cruz-Motta (2007), así como Martín et al. (2007) establecieron que los aportes del Río Orinoco introducen cambios en resuspensión, salinidad y distribución de los nutrientes en las aguas del Océano Atlántico venezolano y el Mar Caribe, los cuales pudieran ser los responsables de la distribución de A. marginatus en la ecorregión, y las variaciones de éstos, los causantes de las diferencias en la misma durante las épocas muestreadas. Ya que estos factores tal vez podrían limitar la supervivencia de los organismos a partir de cierta talla, por causa del estrés generado debido a la poca capacidad de regular los cambios, se explicaría el hecho de que las estrellas de menor tamaño se encontraron en el sector Plataforma Deltana, donde el

CUADRO 6

Valores de similitud obtenidos con el análisis SIMPER para los parámetros estudiados de la dieta de A. marginatus

TABLE 6

Simmilarity values obtained with SIMPER analysis for the different parameters studied for the diet of A. marginatus

$\begin{array}{lccccc}\text { Parámetro evaluado } & \begin{array}{c}\% \\ \text { Similitud }\end{array} & \begin{array}{c}\text { Categoría que } \\ \text { más aporta }\end{array} & \begin{array}{c}\text { Abundancia } \\ \text { Promedio }\end{array} & \begin{array}{c}\text { Similitud } \\ \text { Promedio }\end{array} & \begin{array}{c}\% \\ \text { Contribución }\end{array} \\ 2006 & 28.84 & \text { Bivalvos } & 1.99 & 16.28 & 56.43 \\ 2004-2005 & 19.29 & \text { Bivalvos } & 1.84 & 11.95 & 61.95 \\ \text { Lluvia } & 21.38 & \text { Bivalvos } & 1.66 & 14.76 & 69.04 \\ \text { Sequía } & 20.28 & \text { Bivalvos } & 2.04 & 11.37 & 56.06 \\ \text { Golfo de Paria } & 26.68 & \text { Bivalvos } & 2.44 & 15.73 & 58.95 \\ \text { Boca de Serpiente } & 37.79 & \text { Bivalvos } & 17.60 & 32.86 & 86.95 \\ \text { Plataforma Deltana } & 19.54 & \text { Bivalvos } & 1.33 & 12.22 & 61.30\end{array}$

CUADRO 7

Valores de disimilitud de la dieta de A. marginatus obtenidos con el análisis SIMPER entre las combinaciones de los parámetros estudiados

TABLE 7

Dissimilarity values for the diet of A. marginatus with SIMPER analysis between combinations of the different parameters studied

$\begin{array}{lcccccc}\text { Parámetro evaluado } & \begin{array}{c}\% \\ \text { Disimilitud }\end{array} & \begin{array}{c}\text { Categoría que } \\ \text { más aporta }\end{array} & \begin{array}{c}\text { Abundancia } \\ \text { Promedio grupo 1 }\end{array} & \begin{array}{c}\text { Abundancia } \\ \text { Promedio grupo 2 }\end{array} & \begin{array}{c}\text { Disimilitud } \\ \text { Promedio }\end{array} & \begin{array}{c}\% \\ \text { Contribución }\end{array} \\ \text { BS vs PD } & 89.03 & \text { Bivalvos } & 17.60 & 1.33 & 55.41 & 62.24 \\ \text { GP vs PD } & 80.38 & \text { Bivalvos } & 2.44 & 1.33 & 18.67 & 23.23 \\ \text { GP vs BS } & 84.42 & \text { Bivalvos } & 2.44 & 17.60 & 49.86 & 59.05 \\ \text { Lluvia vs Sequía } & 80.34 & \text { Bivalvos } & 1.66 & 2.04 & 19.38 & 24.13 \\ \text { 2006 vs 2004-2005 } & 79.70 & \text { Bivalvos } & 1.99 & 1.84 & 18.41 & 23.09\end{array}$

BS: Boca de Serpiente; GP: Golfo de Paria; PD: Plataforma Deltana. 
impacto de las aguas del Río Orinoco es mayor, con un limitado desarrollo de tallas grandes en dicho sector. Autores como Zamorano \& LeyteMorales (2009), concluyen de manera general que los cambios observados en la temperatura, salinidad, contenido de oxígeno y transparencia del agua, alteran las densidades poblacionales del erizo D. mexicanum durante las dos épocas del año (seca y lluvias). Sin embargo, se ha demostrado que al menos los cambios en la salinidad por efecto del Río Orinoco afectan sólo el nivel superficial de la columna de agua, no viéndose influenciadas zonas profundas donde se realizaron los muestreos (Martín et al. 2007).

Parámetros Poblacionales: En los muestreos del 2006, se observó un aumento general en la abundancia del megabentos y macrobentos en el sector Golfo de Paria respecto a la encontrada en los muestreos de 2004-2005 (Klein \& Cruz-Motta 2007, Martín et al. 2007), lo que coincide con un aumento de la densidad de A. marginatus. Dentro de las muestras recolectadas durante el periodo 2004-2005, la mayor distribución geográfica y densidad de dicha estrella se encontró en el sector Plataforma Deltana; esta área tiene la mayor cantidad de desembocaduras de caños, los cuales pueden influir en los nutrientes y temperatura entre otros factores, sin embargo, este fue el sector con menor biomasa. Beddingfield \& McClintock (1993) reportan que A. articulatus (Say 1825) se mueve distancias más grandes en búsqueda de alimento en los lugares con menor densidad de presas, mientras que cuando hay una elevada densidad de presas el desplazamiento de las estrellas es menor. Bone et al. (2007) reportan muy bajas densidades de macrobentos en el sector Plataforma Deltana, lo que puede sugerir que lo propuesto por Beddingfield \& McClintock (1993) pudiera ocurrir también para A. marginatus. El sector Golfo de Paria presentó la mayor biomasa de $A$. marginatus, lo que coincide con una alta densidad de organismos macrobentónicos, reportados por Martín et al. (2007), lo que sugiere una relación entre la densidad de posibles presas y la biomasa de la estrella.
Por otra parte, Lemmens et al. (1995) reportan que las preferencias alimenticias para las especies Astropecten zebra (Sladen 1883) y A. velitaris (Martens 1865) influyen en su distribución y que donde abundaba mucho una especie, la otra estaba ausente. Por esto, la competencia interespecífica podría ser un factor influyente en la distribución de las especies de este género. Este factor también podría estar afectando en el Atlántico venezolano ya que aquí han sido reportadas especies de asteroideos con dietas similares al organismo de estudio, como lo son A. articulatus, Luidia alternata (Say 1825) y Luidia senegalensis (Lamarck 1816) (Klein \& Cruz-Motta 2007, Martín et al. 2007), que se encontraron en muchas de las estaciones donde no estaba presente A. marginatus.

La tendencia general de la mayoría de las estaciones muestreadas fue a una disminución de la densidad de estrellas en la época de lluvia. La prueba Chi cuadrado muestra que la diferencia en la distribución de densidades por época no es significativa en toda la zona de estudio, aún cuando al evaluar los sectores por separado si da una diferencia significativa. Esta posible contradicción puede que se deba a una compensación en los datos numéricos con tendencias opuestas entre el sector Golfo de Paria y Plataforma Deltana.

En la época de lluvia se produce un aumento en el caudal del Río Orinoco, el cual genera un fuerte impacto en toda el área de estudio, trayendo como consecuencias un aumento en el aporte de nutrientes, sedimentación y resuspensión, lo que pudiera ser la causa de la variación en la distribución de la estrella y la disminución de las densidades en la mayoría de las estaciones. Este río presenta un ciclo estacional de ascenso y descenso, con un máximo de descarga en agosto y un mínimo en marzo, durando el periodo de ascenso unos cinco meses y el de descenso unos siete meses (Martín et al. 2007). En el Golfo de Paria se observa un aumento en la salinidad a nivel de fondo en la época de sequía, así como una disminución en los niveles de Nitrógeno total. En los sectores Boca de Serpiente y Plataforma 
Deltana, por ser sectores con mayores profundidades no se observaron cambios marcados en los parámetros oceanográficos a nivel del fondo entre las dos épocas (Martín et al. 2007).

En cuanto a la relación tamaño-peso de $A$. marginatus, se observó que era proporcional, a medida que aumenta el tamaño de la estrella aumenta su peso, influyendo en esta variación la cantidad de alimento que haya ingerido la estrella, lo que concuerda con lo reportado por Ribi \& Jost (1978) para A. aranciacus (Linnaeus 1758).

En estudios anteriores sobre A. marginatus en Venezuela, Bitter (1984) reporta tamaños entre $20-44 \mathrm{~mm}$, con un promedio de $31.68 \pm 4.49 \mathrm{~mm}$; el cual es el intervalo de talla más abundante en las estrellas de este estudio. Sin embargo, las dimensiones encontradas abarcan un intervalo de tallas mayor (7-76mm). Por otra parte, Scheibling (1980a, 1980b) reportó que el crecimiento de los asteroideos es principalmente dependiente de la cantidad y calidad del alimento. Por lo tanto, la distribución de frecuencia de tallas refleja la disponibilidad de alimento más que la estructura etaria de la población, dado que la mayor proporción de individuos de mayor tamaño se encuentra donde la densidad de alimento es mayor. Esto podría marcar la diferencia entre las tallas previamente reportadas para la especie y las encontradas en la ecorregión Delta del Orinoco.

Dieta: Se observó que los principales organismos ingeridos por A. marginatus fueron en cuanto a frecuencia numérica, los moluscos, principalmente bivalvos y gasterópodos, similar a lo encontrado por Bitter $(1984,2000)$ en Golfo Triste, Venezuela y en Brasil por Vala (2005) y Caregnato et al. (2009). Estos organismos también han sido reportados como los principales componentes de la dieta de otras especies del género Astropecten (Wells et al. 1961, Ribi et al. 1977, Bitter et al. 1980, Bitter \& Penchaszadeh 1983, Ganmanee et al. 2003, Wells \& Lalli 2003).

Del análisis del contenido estomacal de A. marginatus recolectadas durante el 2006 en el Golfo de Paria, se observó una diferencia notoria en la abundancia de ostrácodos entre la época de lluvia (18.9\%) y sequía (34.5\%). Una de las posibles causas del aumento de ostrácodos en la dieta es que sea un aumento general de la disponibilidad del recurso, ya que otros componentes incluyendo los bivalvos y gasterópodos también aumentaron su abundancia, aunque no en las mismas proporciones. Lamentablemente, la abundancia de esta clase nunca ha sido evaluada en el área. Cabe acotar que la frecuencia de aparición de los ostrácodos en ambas épocas fue similar, y que el aumento de los mismos en época de sequía no implicó una disminución del porcentaje de abundancia de bivalvos y gasterópodos en la dieta. Caregnato et al. (2009), encontraron que entre los meses de octubre a diciembre se observó un mayor número de presas consumidas que en el periodo mayo-junio, planteando que los cambios temporales en la disponibilidad de presas tienen una fuerte influencia en la dieta de $\mathrm{A}$. brasiliensis Müller \& Troschel 1842. Ellos plantean que los cambios de temperatura del agua en las diversas estaciones pueden ocasionar cambios en las tasas de alimentación de las estrellas, sin embargo las mediciones de temperatura realizadas en la ecorregión Delta del Orinoco no muestran grandes diferencias en la temperatura del agua entre las dos épocas muestreadas.

Al comparar los componentes alimenticios encontrados en el contenido estomacal de $A$. marginatus con lo reportado en las mismas épocas y sectores para el macrobentos presente, se pudo observar una diferencia notable entre los elementos más abundantes del macrobentos con los del contenido estomacal de las estrellas en cada sector, ya que en este componente los organismos más abundantes reportados independientemente de la época, año o sector muestreado fueron los crustáceos y poliquetos, ocupando los moluscos frecuencias inferiores al 7\% (Klein \& Cruz-Motta 2007, Martín et al. 2007). La baja densidad de bivalvos y gasterópodos en la infauna del lugar demuestra una preferencia alimenticia por los moluscos, así como lo establecen Lera (1980), Bitter (1984), Wells \& Lalli (2003) y Caregnato et al. (2009), 
posiblemente debido a la facilidad de captura de los organismos con conchas. Entre los equinodermos, las estrellas de mar juegan un papel importante como depredadores de una variedad de organismos macrobentónicos, en especial moluscos, sobre los cuales ejercen una influencia importante en la estructura de la comunidad de las presas (Caregnato et al. 2009).

Además, los componentes de la dieta encontrados en el contenido estomacal de A. marginatus reflejan un hábito alimenticio carroñero-detritívoro, además de depredador, lo que explicaría la abundancia de escamas, restos óseos y calcáreos presentes, así como de otros componentes como pellets fecales. Esto mismo se ha encontrado en A. articulatus, donde Bitter \& Penchaszadeh (1983) expresan que puede ser en alguna medida carroñera-detritívora, explicando así la presencia de arena, restos de conchas de bivalvos, espinas de erizos, pequeños trozos de coral, etc. en sus contenidos estomacales. Sin embargo, otra posibilidad es que haya ocurrido una ingesta accidental, al tratar de capturar pequeños organismos del macrobentos, posibilidad también planteada por Bitter (2000). El método de captura utilizado en este estudio, pudo haber ocasionado un aumento de la aparición de elementos como el sedimento y otras partículas encontradas en la superficie del sustrato, los cuales pueden ser introducidos por la boca al ser arrastrados los organismos del megabentos junto con el sedimento y demás componentes del sustrato.

Bitter (1984) plantea que el sedimento encontrado dentro del contenido estomacal de A. marginatus y el detritus podría ser explicado por los organismos detritívoros consumidos por la estrella. Sin embargo, la cantidad de sedimento encontrada dentro del contenido estomacal de algunas estrellas, que en algunos casos se observó estómagos completamente llenos sólo de sedimento, no parece corresponder con la poca cantidad de sedimento que pudiese aportar un organismo detritívoro consumido por esas estrellas, ya que siempre fueron encontrados de pequeños tamaños.

La ingesta de equinodermos no ha sido reportada para A. marginatus, sin embargo, sí es un componente importante dentro de la dieta de otros asteroideos (Wells et al. 1961, Penchaszadeh \& Lera 1983, Wells \& Lalli 2003, Gammanee et al. 2003). En el presente estudio se encontraron pocos ejemplares de equinodermos (un pepino y 12 juveniles de asteroideos). La presencia de juveniles de asteroideos en el contenido estomacal de algunas estrellas sugiere también una posibilidad de canibalismo en la especie, pero dada la forma de colecta no se puede asegurar este hábito ya que durante el proceso de arrastre los juveniles pudieron ser forzados a entrar por la boca de las estrellas.

En general, se ha catalogado a las estrellas de este género como depredadores voraces y oportunistas, siendo posible encontrar en sus estómagos una amplia variedad de presas, con una preponderancia de organismos de la infauna (Bitter 1984, Wells \& Lalli 2003), tal como fue observado en este estudio.

Gammanee et al. (2003) reportan que $A$. scoparius (Müller \& Troschel 1842), no presenta diferencias alimenticias entre clases de talla de las estrellas. Sin embargo, se ha planteado que las estrellas presentan una búsqueda activa de la presa y son capaces de excavar en el sustrato hasta conseguirla, presentando también selectividad en el tamaño de la presa, prefiriendo los organismos de tallas más pequeñas (Lera 1980, Bitter \& Penchaszadeh 1983, Beddingfield \& McClintock 1993, Vala 2005, Caregnato et al. 2009). Lera (1980) reporta un intervalo de tallas de presas para A. marginatus de 0.5 a $10 \mathrm{~mm}$, similar a las dimensiones encontradas para la mayoría de los elementos alimenticios encontrados en este estudio. Caregnato et al. (2009) reportan que no se observó relación entre el tamaño de la estrella y la cantidad y tamaño de las presas que puede ingerir, similar a lo encontrado en este estudio para A. marginatus.

Finalmente, se puede concluir que A. marginatus es una estrella de amplia distribución geográfica en la zona muestreada, la cual varía tanto espacial como temporalmente a lo largo del área de estudio, así como en las profundidades muestreadas; siendo de pequeñas dimensiones (hasta $7.6 \mathrm{~cm}$ ) y se alimentan de una gran 
variedad de organismos, pero principalmente de bivalvos, prefiriendo presas de pequeñas dimensiones.

\section{RESUMEN}

Astropecten marginatus Gray 1840 presenta una amplia distribución en el norte y este de sur América, encontrándose en fondos arenosos y fangosos de aguas someras y profundas. Se determinó su distribución, parámetros poblacionales tales como: talla, peso y dieta, de los ejemplares provenientes de los muestreos de megabentos de los proyectos LBAPD y CFI en las épocas de lluvia y sequía en la ecorregión Delta del Orinoco, Venezuela. Se dividió la ecorregión en tres sectores: Golfo de Paria, Boca de Serpiente y Plataforma Deltana. Las estrellas fueron medidas, pesadas y se extrajo su estómago para la determinación de las presas. Las mayores densidades se encontraron en época de sequía en Golfo de Paria (2006) y en época de lluvia en Plataforma Deltana (2005). El intervalo de tallas más frecuente fue $3 \cdot 1-4.6 \mathrm{~cm}$. La mayor biomasa se encontró en Golfo de Paria en época de sequía. No se observaron relaciones entre el tamaño de la estrella y la profundidad de muestreo. Existe una dominancia de los moluscos en la dieta, a pesar de tener una alimentación heterogénea. Se observó que aparte de la depredación, también pueden presentar comportamientos carroñeros y detritívoros.

Palabras clave: Astropecten marginatus, Echinodermata, Venezuela, megabentos, Astropectinidae, dieta, distribución.

\section{REFERENCIAS}

Beddingfield, S.D. \& J.B. McClintock. 1993. Feeding behavior of the sea star Astropecten articulatus (Echinodermata: Asteroidea): an evaluation of energy-efficient foraging in a soft-bottom predator. Mar. Biol. 115: 669-676.

Benavides-Serrato, M., G. Borrero-Pérez, O. Solano \& G. Navas. 2005. Listado taxonómico de los asteroideos (Echinodermata: Asteroidea) de la plataforma y el talud superior del Caribe colombiano. Rev. Biol. Trop. 53: 171-194.

Bitter, R. 1984. Composición de la dieta de Astropecten marginatus (Echinodermata: Asteroidea). Bol. Inst. Oceanogr. Ven. U. Oriente 23: 169-176.

Bitter, R. 2000. Nicho complementario en tres especies de Asteroideos del género Astropecten. CROIZATIA 1: 31-37.
Bitter, R., R. Molinet \& P. Penchaszadeh. 1980. Interacción trófica entre dos estrellas de mar (Astropecten riensis y Tethyaster vestitus) en Golfo Triste, Venezuela. Bol. Inst. Oceanogr. S. Paulo 29: 61-63.

Bitter, R. \& P. Penchaszadeh. 1983. Ecología trófica de dos estrellas de mar del género Astropecten coexistentes en Golfo Triste, Venezuela. Stud. Neotrop. Fauna Environ. 18: 163-180.

Bone, D., I. Chollett \& C.T. Rodríguez. 2007. Macrobentos de aguas profundas en la costa atlántica venezolana. Interciencia 32: 477-481.

Caregnato, F.F., F. Wiggers, C. Tarasconi \& L. Veitenheimer-Mendes. 2009. Taxonomic composition of mollusks collected from the stomach content of Astropecten brasiliensis (Echinodermata:Asteroidea) in Santa Catarina, Brazil. R. Bras. Bioci. 7: 252-259.

Clark, A. \& M. Downey. 1992. Starfishes of the Atlantic. Chapman \& Hall Identification Guides, 3. Chapman \& Hall, London, United Kingdom.

Clarke, K.R. \& R.N. Gorley. 2006. Primer v6: User Manual/Tutorial. PRIMER-E, Plymouth, United Kingdom.

Ganmanee, M., T. Narita, S. Lida \& H. Sekiguchi. 2003. Feeding habits of asteroids, Luidia quinaria and Astropecten scoparius, in Ise Bay, Central Japan. Fish. Sci. 69: 1121-1134.

Hendler, G., J. Miller, D. Pawson \& P. Kier. 1995. Sea stars, sea urchins, and allies. Smithsonian Institution, Washington D.C., USA.

Herrera-Moreno, A. \& L. Betancourt. 2004. Especies de equinodermos recientes (Echinodermata: Crinoidea, Asteroidea, Ophiuroidea, Echinoidea y Holothuroidea) conocidas para la Hispaniola. Rev. Cien. Soc. U. INTEC 29: 506-533.

Kenk, R. 1944. Ecological observations on two puertorican echinoderms, Mellita lata and Astropecten marginatus. Biol. Bull. 87: 177-187.

Klein, E. \& J.J. Cruz-Motta. 2007. Plan de monitoreo de los posibles impactos del proyecto Corocoro etapa I. Informe final. INTECMAR-USB, Caracas, Venezuela.

Krebs, C. 1998. Ecological methodology. Addison-Wesley, Menlo Park, California, USA.

Lemmens, J.W.T., P.W. Arnold \& A. Birtles. 1995. Distribution patterns and selective feeding in two Astropecten species (Asteroidea: Echinodermata) from Cleveland 
Bay, Northern Queensland. Mar. Freshwater Res. 46: 447-455.

Lera, M. 1980. Repartición de recursos entre dos estrellas de mar, Astropecten marginatus (Gray, 1840) y Luidia senegalensis (Lamark) coexistentes en aguas someras del Parque Nacional Morrocoy (estado Falcón, Venezuela). Tesis de grado, Universidad Simón Bolívar, Caracas, Venezuela.

Martín, A., L. Malavé, D. Sánchez, R. Aparicio, F. Arocha, D. Bone, J.A. Bolaños, J. Bolaños-Jiménez, J. Castañeda, J.J. Cárdenas, A.K. Carbonini, Y.J. Díaz, H.J. Guada, E. Klein, R. Lazo, A. Lemus, M. Lentino, C. Lira, C. Lodeiros, R. López, B. Marín, G. Martínez, B. Márquez, A. Márquez, R. Molinet, F. Morales, J. Posada, A. Prieto, A. Riera, C.T. Rodríguez, A. Ramírez, W. Senior, P. Solana, H. Severeyn, P. Spiniello, E. Valera, C. Yanes \& E. Zoppi. 2007. Línea Base Ambiental Plataforma Deltana. In A. Martín \& D. Bone (eds.). Petróleos de Venezuela, S.A., Universidad Simón Bolívar, Caracas, Venezuela.

Miloslavich, P., E. Klein, A. Martín, C. Bastidas, B. Marín \& P. Spiniello. 2005. Venezuela, p. 109-136. In P. Miloslavich \& E. Klein (eds.). Caribbean Marine Biodiversity the known and the unknown. DEStech, Pennsylvania, USA.

Miloslavich, P. \& E. Klein. 2008. Ecorregiones marinas del caribe venezolano, p. 16-19. In E. Klein (ed.). Prioridades de PDVSA en la conservación de la biodiversidad en el caribe venezolano. Petróleos de Venezuela, S.A. - Universidad Simón Bolívar- The Nature Conservancy, Caracas, Venezuela.

Penchaszadeh, P. \& M. Lera. 1983. Alimentación de tres especies tropicales de Luidia (Echinodermata: Asteroidea) en Golfo Triste, Venezuela. Carib. J. Sci. 19: $1-2$.

Ribi, G., R. Scharer \& P. Ochsner. 1977. Stomach contents and size-frequency distributions of two coexisting sea star species, Astropecten aranciacus and $A$. bispinosus, with reference to competition. Mar. Biol. 43: 181-185.

Ribi, G. \& P. Jost. 1978. Feeding rate of sea stars related to prey density. Mar. Biol. 45: 249-254.

Scheibling, R. 1980a. Abundance, spatial distribution, and size structure of populations of Oreaster reticulatus (Echinodermata: Asteroidea) on sand bottoms. Mar. Biol. 57: 95-105.

Scheibling, R. 1980b. Abundance, spatial distribution, and size structure of populations of Oreaster reticulatus (Echinodermata: Asteroidea) on sand bottoms. Mar. Biol. 57: 107-119.

Sturges, H. 1926. The choice of a class-interval. J. Amer. Statist. Assoc. 21: 65-66.

Vala, L. 2005. Análise do conteúdo estomacal de duas espécies de estrela-do-mar: Astropecten marginatus (Gray, 1840) e Luidia senegalensis (Lamarck, 1816) na Enseada da Caraguatatuba, SP sudeste do Brasil. Tesis de grado, Centro Universitário da Fundação de Ensino Octávio Bastos, São João da Boa Vista, Brasil.

Wells, H.W., M.J. Wells \& I.E. Gray. 1961. Food of the sea star Astropecten articulatus. Biol. Bull. 120: 265-271.

Wells, H. \& C.M. Lalli. 2003. Astropecten sumbawanus (Echinodermata: Asteroidea) in Withnell Bay, northwestern Australia, p. 209-216. In F.E. Wells, D.I. Walker \& D.S. Jones (eds.). The Marine Flora and Fauna of Dampier, Western Australia. Western Australian Museum, Perth, Australia.

Zamorano, P. \& G.E. Leyte-Morales. 2009. Equinodermos asociados a formaciones arrecifales en Zihuatanejo y Acapulco, Guerrero, México. Bol. Invest. Mar. Cost. 38: 7-28.

Zoppi de Roa, E. 1967. Contribución al estudio de los equinodermos de Venezuela. Acta Biol. Ven. 5: 267-333. 